\title{
Testicular Tuberculosis in an HIV Positive Patient Co-infection with Pulmonary Tuberculosis Mimicking Malignancy: A Case Report
}

\author{
Wahyu Agung Purnomo ${ }^{1,2^{*}}$, Tutik Kusmiati², Soedarsono \\ ${ }^{1}$ Dr. Soebandi General Hospitasl, Jember, Indonesia. \\ ${ }^{2}$ Department of Pulmonology and Respiratory Medicine, Faculty of Medicine, Universitas Airlangga/Dr. Soetomo \\ General Hospital, Surabaya, Indonesia.
}

\section{ARTICLE INFO}

\section{Article history:}

Received 29 April 2020

Received in revised form 26 May

2020

Accepted 27 May 2020

Available online 30 May 2020

\section{Keywords:}

Testicular mass,

Orchitis tuberculosis,

HIV positive,

Pulmonary tuberculosis.

\begin{abstract}
Background: Extrapulmonary involvement can be seen in more than 50\% of patients with concurrent HIV and tuberculosis (TB). Approximately $7 \%$ of those are presented as a rare case, namely testicular TB, with scrotal mass as common clinical manifestation. Testicular TB should be a different diagnosis, especially in areas with a high prevalence rate for $\mathrm{TB}$.

Case: A 24-year-old male with HIV positive co-infection with pulmonary TB presented with a progressively increasing painless right testicular swelling of 1-month duration. History of chronic cough was complained, accompanied by loss of appetite and decrease of body weight. There was no history of testicular traumatic accident before. Genital examination was right testicular mass measuring $7 \mathrm{~cm} \mathrm{x} 4 \mathrm{~cm}$ and depletion of right side scrotal skin with pus and necrotic tissue. He had right orchidectomy by an urologist in a private hospital; then an anatomical pathology was examined. The result of the microscopic evaluation showed granuloma inflammation process typical for orchitis tuberculosis. The patient was reactive for HIV and had Mycobacterium tuberculosis (MTB) detected, Rifampicin resistant not recognized as the result of Gene Xpert MTB/RIF assay.

Conclusion: Although a rare case, testicular TB should be a different diagnosis when there is a patient with HIV positive concurrent pulmonary TB with scrotal mass as clinical manifestation, especially in areas with a high prevalence rate for TB.
\end{abstract}

\section{INTRODUCTION}

Extrapulmonary tuberculosis (TB) has become more common since the advent of Human Immunodeficiency Virus (HIV) infection. It has been reported that $50 \%$ of patients with concurrent HIV and TB develop extrapulmonary $\mathrm{TB}^{1}$. Approximately $7 \%$ of those are presented as a rare case, namely testicular TB. Patients with HIV infection present an increased risk of genitourinary TB. ${ }^{2}$ Genitourinary involvement can be found in extensive pulmonary tuberculous disease through hematogenous spreading or present as a primary genital lesion as a consequence of tubercular lesions in the prostate or kidneys. Nevertheless, it is still a controversy about which of these two pathways is the more common. ${ }^{34}$

Testicular TB should be a different diagnosis other than the testicular tumor, acute infection, infarction, and granulomatous orchitis when there is a patient with HIV positive concurrent pulmonary TB with scrotal mass as clinical manifestation, especially in areas with a high prevalence rate for TB. ${ }^{5}$

\section{CASE}

A 24-year-old male with HIV positive coinfection with pulmonary TB presented with a progressively increasing painless right testicular swelling of 1-month duration. History of chronic cough was complained, accompanied by loss of appetite and decrease of body weight. There was no history of traumatic testicular accident before. The retrograde investigation found some of risk factors of HIV transmissions, such as the background of occupations and high risk of sexual activities.

Genital examination was right testicular mass measuring $7 \mathrm{~cm} \times 4 \mathrm{~cm}$ and depletion of right-side scrotal skin with pus and necrotic tissue (Figure 1). He had right orchidectomy by an urologist in a private hospital; then an anatomical pathology was examined. It 


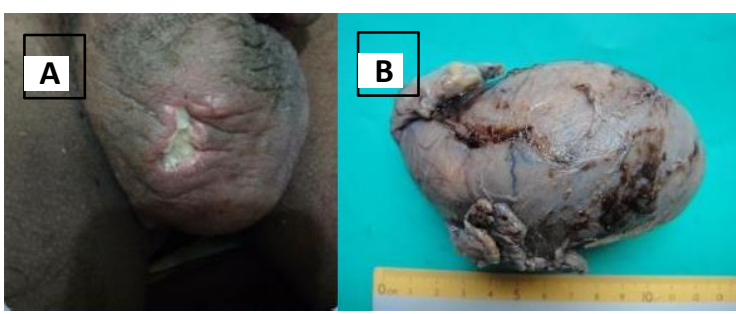

Figure 1. A. Swelling and scrotal skin of testicular TB

B. Testicular tissue size $7 \times 4 \times 4 \mathrm{~cm}$, with smoothouter surface, on the slices appear a gray-white mass. The consistency is soft and the boundaries are not clear
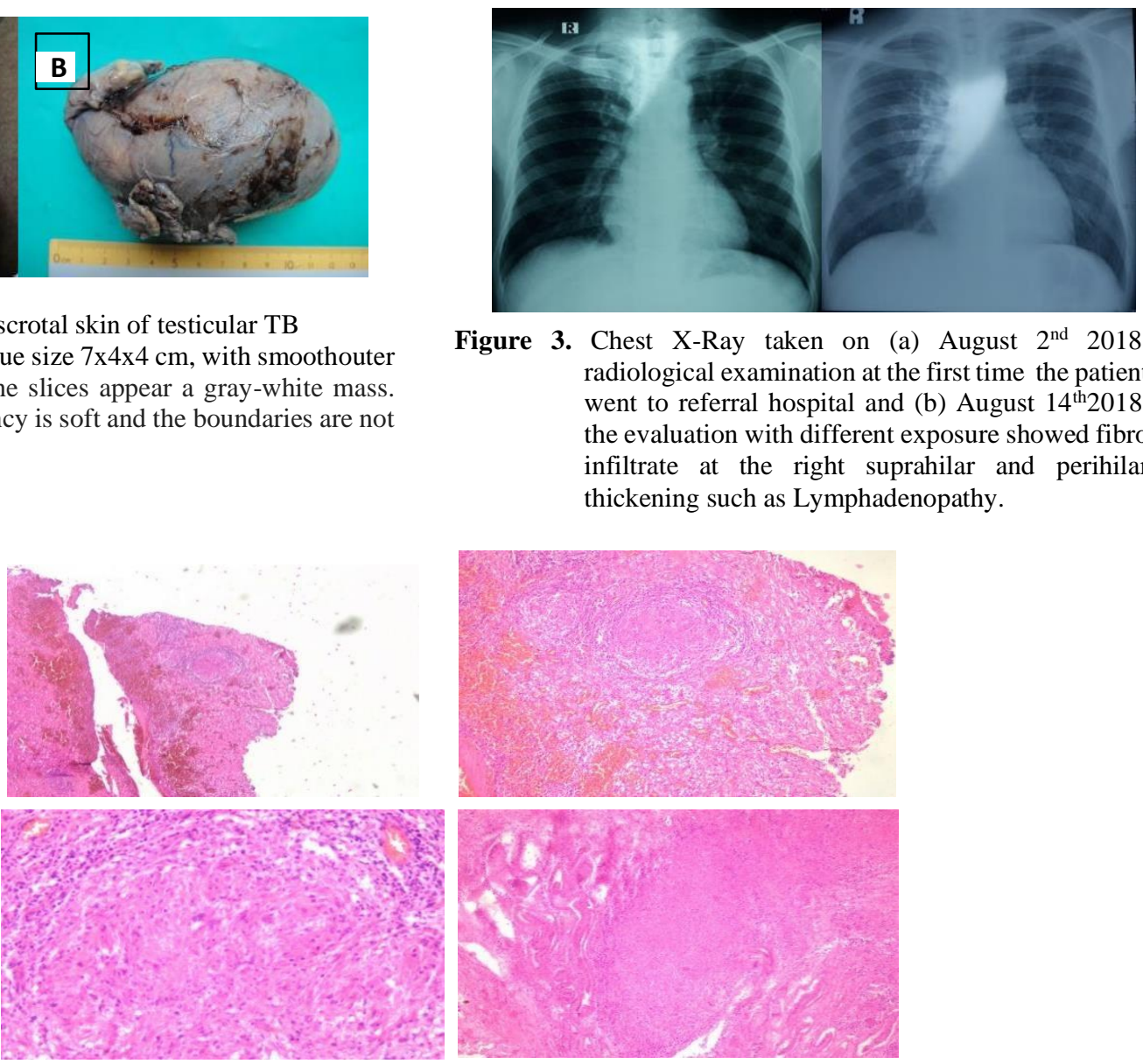

Figure 3. Chest X-Ray taken on (a) August $2^{\text {nd }} 2018$, radiological examination at the first time the patient went to referral hospital and (b) August $14^{\text {th }} 2018$, the evaluation with different exposure showed fibro infiltrate at the right suprahilar and perihilar thickening such as Lymphadenopathy.
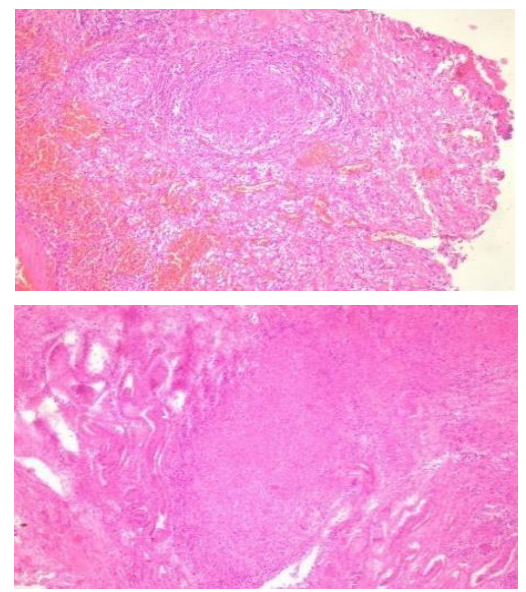

Figure 2. Histopathological result: Indicates pieces of testicular tissue. It appears that the distribution of epithelioid histiocytes cells form granulomas. There is also the distribution of inflammation cells of neutrophils, lymphocytes, and plasma cells.

demonstrated pieces of testicular tissue, as well as lymphocytic inflammatory cells, plasma cells, several Datia Langhans cells, epithelioid cell groups forming granuloma structures, and areas of pursuit necrosis. In the epididymis, a lymphocytic inflammatory cell was obtained, and there was no sign of malignancy. The conclusion was granulomatous suppurative inflammation process typical for orchitis TB (Figure 2).

Three methods of anti-HIV examinations, all reagents (SD, VIKIA, and FOKUS) were reactive. Besides from the clinical, the diagnose of pulmonary TB were based on the radiological and bacteriological examination. The chest X-ray showed fibro infiltrate at the right suprahilar and perihilar thickening such as lymphadenopathy (Figure 3). The result of two acid-fast bacillus (AFB) tests from sputum was 1+.Mycobacterium tuberculosis (MTB) was low detected, and Rifampicin resistant was not detected from the result of Gene Xpert MTB/RIF assay.

Before starting the anti-tuberculosis drugs (ATD), a high level of the liver function test result was found. Then USG abdomen was performed; the result was decreased echo parenchyma liver intensity could be a process of reactive hepatitis. Viral hepatitis examination was performed, HbsAg and anti $\mathrm{HCV}$ to exclude the cause of increasing aspartate aminotransferase (AST) and alanine aminotransferase (ALT), the results were nonreactive. Non-hepatotoxic

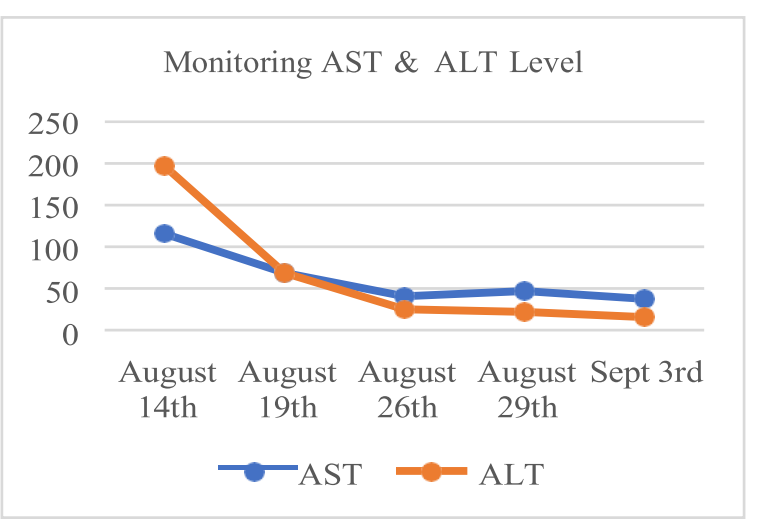

Figure 5. Monitoring AST \& ALT level starting from August $14^{\text {th }}$ until September $3^{\text {rd }} 2018$ decreasing gradually as consideration for ATD regimen selection

drugs were given first (Streptomycin, Ethambutol, and Levofloxacin) and made continues evaluation about the level of AST and ALT (Figure 4). After the level of AST and ALT became normal gradually, Rifampicin, Isoniazid, and Pyrazinamide were reintroduced. Finally, $1^{\text {st }}$ category of ATD was given with extended continuous phase until ten months. After two weeksadministered of ATD, there were not any side effects, and the CD4 count result was 122 cells/uL. Anti-retroviral therapy (ART) was given for the patient. 


\section{DISCUSSION}

TB has become a common opportunisticinfection because of HIV pandemic. HIV infection was arisk factor for the development of TB through the mechanism of reactivating latent infection, a rapid progression in primary infection, or reinfection with MTB. HIV patients had a 20-37 fold chance of having

TB compared to people who do not have HIV infection. ${ }^{5}$ The patient in this case is positive for HIV.

The incidence of extrapulmonary TB has correspondingly increased, and the genitourinary system is the most commonly affected site. Epididymitis is the most common symptom of genitourinary TB in male patients. ${ }^{4}$ Genitourinary system has been reported up to $20 \%$ of pulmonary TB cases. ${ }^{6}$ The manifestation of the patient in this case is orchitis TB. Based on a study by Farer, Lowell, and Meador about extrapulmonary TB in the United States, the prevalence of genitourinarysystem manifestation was $2.34 \%$. Orchitis is one of them, for about $0.09 \% .^{7}$ The patient in this case is a $24-$ year-old male that is included in the age range incidence of testicular TB, 20 until 70 years old. Symptom ofpainless right testicular swelling complained about 1 month was similar to the length of history range basedon a series of Ferrie and Roundle, 1 week until 8 weeks.

The underlying mechanism of testicular TB in the patient can be explained through the pathogenesis process from the onset of infection in the lungs then spread to the genitourinary system. MTB is infected through droplet nuclei of patients with active pulmonary TB disease. These bacteria come through the airwaysand eventually form the primary effect causing lymphangitis and lymphadenopathy in the hilum. So thatthe primary focus (Ghon focus) is built. Then MTB is disseminated hematogen through the bloodstream from the primary focus of the lung to genitourinary tissues causing infection in the kidney, epididymis, testis, prostate, and seminal vesicles. Nevertheless, retrograde extension from prostate and seminal vesicles to the epididymis and testicles may also occur. ${ }^{8}$ The primary focus with necrotic areas from the epididymis extends to testicle. ${ }^{9}$ Primary lesions cause granulomas which are dormant in that lesions for decades and it can be reactivated again. After initial exposure, MTB lives inside the macrophage and begins to replicate slowly. MTB can be reactivated again for several years andcause the inflammatory response and may turn the formation of giant cell or Langhans. The response to reactivation of MTB depends on the patient's immunological. ${ }^{10}$ The patient in this case had the immunocompromised cause of HIV infection. Some of the probabilities of the pathway from infection to the disease of testicular TB for the patient are through the mechanisms of reactivating latent infection, a rapid progression in primary infection or reinfection with MTB.

The weakness of this case report is that invasive action is taken in the form of an orchidectomy at the beginning of the diagnosis process before going through several investigations which are considered to have high accuracy in the case of testicular TB namely testicular ultrasound. ${ }^{11}$ Given the difficulty of establishing a diagnosis of testicular TB, often invasive measures such as orchidectomy are performed. The administration of ATD, such as chemotherapy, can provide a good response if the diagnosis of testicular tuberculosis can be established earlier.

Chemotherapy is the first form of treatment, complete resolution with chemotherapy is rare if caseating destructive lesions have formed before its use, and epididymectomy and orchiectomy may be necessary. ${ }^{12}$ Patients with and without HIV infection have similar response to antituberculous therapy, but sometimes adverse drug reactions occur more commonly to those with HIV infection. The higher risk of relapse for patients with HIV is still unclear. ${ }^{1}$ Along with the treatment in the intensive phase, the patient in this case did not complain about any side effect of ATD.Initial recommended treatment for all forms of extrapulmonary TB unless the patients are known or suspected to be resistant to first-line-drugs are using six-to nine-month regimen (two months of isoniazid [INH], rifampin [Rifadin], pyrazinamide, and ethambutol [Myambutol], followed by four to seven months of isoniazid and rifampin). The level of AST and ALT of the patient were higher than normal before the treatment of ATD. Nonhepatotoxic drugs (Streptomycin, Levofloxacin, and Ethambutol) were given first while monitoring the AST and ALT level. When the AST and ALT level became normal gradually, Rifampicin, Isoniazid, and Pyrazinamide were reintroduced. Finally, $1^{\text {st }}$ category of ATD was given with extended continuous phase until ten months.

ARV can reduce the rate up to $90 \%$ at the individual level and $60 \%$ at the population level, aside from minimizing TB recurrence by $50 \%$. On HIV testing for TB patients who give positive results, recommendations to use ARV therapy are: Start ARV therapy as soon as possible after TB therapy can be tolerated, as soon as two weeks and no more than eight weeks, regardless of CD4 count. The patient was given ARV 2 weeks after ATD therapy.

\section{CONCLUSION}

Although a rare case, testicular TB should be a different diagnosis when there is a patient with HIV positive concurrent pulmonary TB with scrotal mass as clinical manifestation, especially in areas with a high prevalence rate for tuberculosis.

\section{REFERENCES}

1. Golden MP, Vikram HR. Extrapulmonary Tuberculosis:An Overview. Am Fam Physician. 2005;72(9):1761-1768.

2. Ojo BA, Ogwuche EI, Duduyemi BM, Okani C, Umobong EO, Jombo GTA. Testicular Tuberculosis in an HIV Positive Patient Mimicking Malignancy: A Case Report. African J Urol. 2014;20(1):28-30. doi:10.1016/j.afju.2013. 
11.003

3. Chirindel A, Martinez F, Gagliardi JA, Armm MF. Testicular Tuberculosis without Epididymitis Simulating Neoplasm. Radiol case reports. 2015;3(3):133. doi:10.2484/rcr.v3i3.133

4. Ferrie BG, Rundle JSH. Tuberculous Epididymo-orchitis. A Review of 20 Cases. Br J Urol. 1983;55(4):437-439. doi:10.1111/j.1464-410X.1983.tb03340.x

5. Wijaya IMK. Infeksi HIV (Human Immunodeficiency Virus) pada Penderita Tuberkulosis. Semin Nas FMIPA UNDIKSHA III. 2013;3:295-303. http://ejournal.undiksha.ac.id/index.php/semnasmipa/articl e/view/2721/2301

6. Farer LS, Lowell AM, Meador MP. Extrapulmonary Tuberculosis in the United States. Am J Epidemiol. 1979;109(2):205-217. doi:10.1093/oxfordjournals.aje.a112675

7. Muttarak M, Peh WCG. Case 91: Tuberculous Epididymoorchitis. Radiology. 2006;238(2):748-751. doi:10.1148/radiol.2382031851
8. Jaffar A, Mehta JB, Godfrey JH. Tuberculous Epididymoorchitis and Granulomatous Prostatitis Mimicking Neoplasia. J Tenn Med Assoc. 1990;83(12):605-606.

9. Tessler FN, Tublin ME, Rifkin MD. US Case of the Day. Tuberculous Epididymoorchitis. Radiographics. 1998;18(1):251-253. doi:10.1148/radiographics.18.1.9460131

10. Wise GJ, Marella VK. Genitourinary Manifestations of Tuberculosis. Urol Clin North Am. 2003;30(1):111-121. doi:10.1016/s0094-0143(02)00123-4

11. Muttarak M, Peh WCG, Lojanapiwat B, Chaiwun B. Tuberculous Epididymitis and Epididymo-orchitis. Am J Roentgenol. 2001;176(6):1459-1466. doi:10.2214/ajr.176.6.1761459

12. Reeve HR, Weinerth JL, Peterson LJ. Tuberculosis of Epididymis and Testicle Presenting as Hydrocele.Urology. 1974;4(3):329-331. doi:10.1016/0090- 4295(74)90389-6 